\title{
El Mito de la Calidad en la Educación Superior: Colonización de la Esfera de lo Privado en lo Público
}

\author{
Rosa María Ramírez Martínez \\ Facultad de Ciencias de la Conducta-UAEM
}

Resumen: El artículo presenta una tesis central en torno a las iniciativas que en el sector educativo se han dado en nombre de la elevación de la calidad del servicio y de la evaluación: la apuesta por la calidad oculta en realidad una forma de colonización de la esfera de lo privado en lo público; se pretende generar una dinámica más acorde con el sector privado en la educación superior, en detrimento de las funciones sociales que le corresponden.

Abstract: Recently, goverment policies have been oriented to increase the quality levels of educational services in the high superior system, which has included an evaluation era in such institutions. What realy appears trough this policies is a trend to apply the enterprise logic of administration on the wrong site: educational field has a social, public nature and functions, in despite of the private issues.

$\mathbf{P}$

ara comenzar la reflexión acerca del sentido de las políticas públicas para la educación superior, se hace necesario construir algunas categorías fundamentales que posibiliten su comprensión y delimiten la postura teórico-epistemológica que sirva de eje para la aprehensión de tal objeto de conocimiento; de ahí que se parta de las categorías de crisis y estrategias de acumulación para elaborar un contexto interpretativo que dé cuenta de los cambios histórico-sociales que se producen en las relaciones entre las esferas de lo público y lo privado, transformaciones que indican la manera en que se objetivan las negociaciones, luchas, enfrentamientos, resistencias o pactos entre grupos y clases cuya pertenencia y permanencia se relacionan con ambas esferas; en última instancia, se podría indicar que se establecen relaciones entre los sujetos, lo cual permite, entonces, comprender cómo en el diseño de las políticas en general, y específicamente en las políticas educativas, se concretan estas relaciones en un compromiso político y cómo todo esto implica una nueva recomposición del todo social. 


\section{Las estrategias de acumulación y la crisis}

La base histórica o punto de partida de la producción, específicamente capitalista, es la acumulación originaria, que no es más que el proceso mediante el cual son expropiados los productores directos; este modo de producción impulsa y se desarrolla a partir de la acumulación de capital en cuanto proceso de transformación de los medios de producción en capital y de apropiación privada del valor producido sobre la base de la propiedad de dichos medios. En esta forma se entiende que la acumulación en cuanto proceso, consiste en el incremento paulatino del capital mediante la reproducción (Marx, 1978: 607-649).

El proceso capitalista de producción consiste esencialmente en la producción de plusvalor... y su reconversión de una parte del mismo en capital, o sea la acumulación,...es el objetivo directo y el motivo determinante de la producción capitalista. Con esta producción del plusvalor sólo queda concluido el primer acto del proceso capitalista de producción, el proceso directo de producción. Llega entonces el segundo acto del proceso: debe venderse toda la masa mercantil, el producto global...Por ello hay que expandir constantemente el mercado. La desvalorización periódica del capital ya existente, que es un medio inmanente al modo capitalista de producción para contener la baja en la tasa de ganancia y para acelerar la acumulación de valor de capital mediante la formación de capital nuevo, perturba las condiciones dadas dentro de las cuales se lleva a cabo el proceso de circulación y reproducción del capital, por lo cual está acompañada por paralizaciones súbitas y crisis del proceso de producción (Marx, 1978: 312, 313-320).

Si la dinámica básica del sistema está sometida a la lógica de la producción del valor, y si ésta encuentra dificultades, la reproducción del sistema tiende a paralizarse en tanto se procesan los reajustes estructurales adecuados, entonces todo cambio estructural viable debe por lo menos satisfacer una condición necesaria: restaurar las condiciones de una valorización adecuada del capital, es decir, las condiciones en que el proceso de formación de valor es prolongado más allá del simple reemplazo del valor de las materias empleadas y del valor de la fuerza de trabajo, de tal modo que se genere un excedente o plusvalía.

Marx establece no solamente la posibilidad de una acumulación larga y durable, sino... que sugiere y anticipa el tipo de acumulación de la producción en masa, pero no se contenta con establecer esta posibilidad teórica de la acumulación larga o sobre la escala ampliada, enuncia también los obstáculos que se levantan en su camino, siendo la 
acumulación capitalista esencialmente una relación contradictoria, hace siempre posible, la aparición de crisis, en el centro de las cuales se halla la cuestión de la técnica.(Marx, 1994: 26).

Las diferencias o desfases de productividad se manifiestan ya sea por oscilaciones artificiales que se compensan en un plazo breve, ya sea por una acumulación progresiva de las divergencias que, o bien conducen a una crisis, o bien son reconocidas lentamente, como un cambio en las estrategias de acumulación y en las formas de distribución o consumo que terminan por imponerse (Boyer y Coriat, 1985: 9-10).

El desarrollo de las estrategias o regímenes de acumulación implican un reordenamiento estructural que posibilite y estimule el crecimiento de las fuerzas productivas y la posibilidad creciente de la acumulación del excedente o plusvalía. Y esto, a partir de una condición previa: que el reordenamiento sea capaz de "sanear" el funcionamiento del proceso de valorización y de reproducción del capital. La necesidad de sanear no es gratuita, pues el proceso de acumulación se comienza a atascar, se enreda en contradicciones que lo paralizan. En esta forma, los cambios provocan a la vez la cancelación y el surgimiento de un patrón, estrategia o régimen de acumulación diferente, pero que esencialmente se siga manteniendo, aunque varíen las formas de distribución o de consumo a través de las cuales se posibilita la realización del valor generado.

Las contradicciones que se generan en el proceso de acumulación de capital se encubren bajo la instalación de un modelo de desarrollo (Elkan, 1975: 63-64) que se niega a sí mismo; por un lado la masa de valores de uso y de cambio - capital y riqueza social-se acumula por la vía del aumento de plusvalía relativa, es decir, de un progreso técnico que procura, a la vez, la disminución de los costos y la intensificación del uso del capital; pero por el otro lado, en cada nuevo estadio de la acumulación la composición de capital se modifica en detrimento del capital variable, el único que produce plusvalía, y que se intercambia por la fuerza de trabajo (Elkan, 1975: 42-46).

A la forma general de la crisis corresponde la posibilidad de contradicción específica de esa misma forma en un tiempo histórico donde se concreta, engendrando un nuevo sistema de contradicciones que, en primera instancia y para un plazo que no es corto, vuelve a dinamizar el sistema, para posteriormente volverse obsoleto. Así pues, 
el capitalismo nunca funciona en abstracto, la matriz esencial siempre se especifica y particulariza con cargo a cierta modalidad, históricamente determinada.

\section{La cuestión de partida para comprender la crisis política, la discusión de la dicotomía entre lo público y lo privado}

Con las transformaciones de las relaciones de producción en las formas capitalistas, se establece el debate de la gran dicotomía de lo público y lo privado, un debate secular a partir de ciertos criterios: económicos, políticos y legales, que escinden las dos esferas. Los términos público y privado son fundamentales en el lenguaje del derecho, la vida política y social pero son causa de continua confusión; muchas cosas aparecen como públicas y privadas al mismo tiempo en diversos grados y en distintas formas. Difícilmente estos términos pueden ser suprimidos, ya que aportan elementos para la comprensión de la política y de las transformaciones del Estado y de la sociedad en su totalidad.

Como primer intento de reflexión y aproximación al término de lo público a partir del sentido común, se ha denominado como públicas a aquellas organizaciones accesibles a todos en contraposición a sociedades cerradas, así lo público se considera como abierto. Estas organizaciones deben su atributo a su tarea de cuidar del bien común, del interés público o de todo el pueblo; de esta forma, público refiere, en primera instancia, a lo común referido a todos los ciudadanos. Por lo que el ciudadano con espíritu público es aquél que se preocupa por toda la comunidad.

El Estado actúa en nombre de toda la sociedad, por lo menos en teoría, es la administración pública, en este sentido significa lo "oficial"; debe su atributo de lo público a su tarea de cuidar del bien común, de todos los ciudadanos. Bajo la idea de lo común, al ciudadano libre se presenta el Estado como instancia superior, arriba de la sociedad; a éste le toca dirimir sobre los conflictos de la misma y con ello se legitima su intervención en el intercambio mercantil a través de sanciones jurídicas; es en esta relación donde se concreta el vínculo entre lo público y lo privado. Esta aproximación de la esfera de lo público pertenece, específicamente, a la sociedad burguesa, que se asentó como ámbito del tráfico mercantil y del trabajo social según sus propias leyes, que integrarán la esfera de lo privado. Es por ello que de la idea de "lo común arranca una línea hacia el bien común o público enfrentado a lo particular, es lo separado, en un sentido de lo privado 
que se equipara con intereses particulares" (Habermas, 1981: $42-43,45)$, es decir, cuando se generaliza la apropiación privada del excedente.

El Estado liberal tiene varias características que lo distinguen de otros; por ejemplo, el tipo de gobierno público implica una elaborada estructura de reglas que limitan el ejercicio de poder; en segundo lugar, cuando los miembros de una sociedad liberal piensan que sus hogares, negocios, iglesias y otras formas de asociación se encuentran dentro de la esfera privada, están poniendo límites al poder público, tal es el caso específico del insuperable derecho de propiedad privada (Kamerman y Kahn, 1993: 31-32).

Un poco después, las empresas capitalistas comienzan a desprenderse de su inveterada vinculación al reducto de la privacidad, lo cual resulta de las transformaciones en las estrategias de acumulación, en los años treinta, y de una mayor participación de la esfera de lo público en la regulación social con el Estado benefactor; así "el trabajo, la profesión, van adquiriendo una dimensión pública, y aparece el tiempo del ocio como su contrapeso privado. Pero no es poco precaria esa privacidad, completamente invadida como está por la industria de los medios de comunicación" (Domenech (s/f): 21).

Detrás de las categorías legales, aunado a las transformaciones del régimen de acumulación y al papel del Estado, se borran los límites de la dicotomía público y privado: por una parte los intereses privados afectan las políticas del Estado; por la otra, el Estado cruza los límites al regular los contratos privados de las empresas. Así, por medio de las preferencias fiscales y garantías de crédito el Estado moldea la economía privada y la sociedad.

Las crisis son inherentes al capitalismo mismo, debido a las contradicciones y límites que gesta y a la posibilidad de enfrentarlas y de autorregularse por medio del cambio o la modificación del régimen o estrategia de acumulación, es decir, las crisis, generalmente, constituyen soluciones violentas a las contradicciones del desarrollo capitalista y, en tal sentido implican un conjunto de transformaciones en lo económico, pero en ese mismo movimiento pueden traspasar sus efectos a las relaciones del sistema político, generando cambios o reformas en la actuación del Estado, o en lo cultural, modificando valores y formas de identidad; sin embargo, no hay que olvidar que 
ambos sistemas tienen dinámicas particulares y que a su vez generan sus propias paradojas.

En términos generales se puede decir que se reconstruyen relaciones entre la esfera de lo público y lo privado a partir de las crisis. Lo cual significa que las formas de organización política estatal se corresponden con las diversas transformaciones de las estrategias de acumulación; en este sentido, el Estado es expresión de una síntesis de relaciones entre clases y grupos dominantes entre sí y entre clases y grupos dominados, de tal manera que en la esfera de lo público se cristaliza esa síntesis de relaciones y compromisos de clase.

La gran crisis del capitalismo tardío, crisis de régimen de acumulación que se desarrolló en la posguerra y que tenía como estrategia de acumulación el fordismo, es una crisis de carácter mundial que comienza entre 1973 y 1974 y alcanza su plenitud al final de la década de los años setenta y principios de la década de los ochenta. Todo el mundo capitalista cayó en una larga y profunda recesión, combinando por primera vez bajas tasas de crecimiento con altas tasas de inflación. Los patrones y estrategias de acumulación, así como las políticas económicas, se modificaban de acuerdo con las condiciones nacionales, pero teniendo como marco de influencia la propuesta del cambio de paradigma económico para dar salida a la crisis, por el evidente fracaso de la política económica de pleno empleo y de intervención estatal, que caracterizan la estrategia de acumulación fordista (Altvater, 1979: 23-34).

Es la crisis de la década de los setenta, debido al cambio de las estrategias de acumulación, la que se caracteriza por una elevada inflación mundial, por frecuentes choques inducidos por variaciones en los precios externos, menores tasas de crecimiento de los países industrializados, aumento de desempleo e inestabilidad de la balanza de pagos de la mayoría de los países. La recesión en los países industrializados tiene un efecto indirecto adicional sobre la elección de las políticas económicas en los países de Latinoamérica. Esta profunda crisis en la economía internacional acarrea un acelerado deterioro de la situación económica en la mayoría de las naciones.

En el país, el Estado que se deriva de la crisis estructural de la década de los setenta y que se prolonga hasta la siguiente década, tránsito al llamado "neoliberalismo", es entendido como la orientación o el resultado de un conjunto de políticas públicas, frecuentemente 
agrupadas bajo el nombre de "reforma del Estado", que se caracterizan por algunas medidas concretas como son: la privatización de empresas públicas, acompañada de una desregulación más general que implica no sólo la reducción del tamaño del Estado, sino también la injerencia del mismo en una serie de actividades sociales y económicas en las que, durante las etapas anteriores del desarrollo se acostumbró a intervenir (Varela, (s/f): 97-107). Se "adelgaza" alegando que es necesario sanear las finanzas públicas, deja la inversión social en manos del sector privado, reduce sus inversiones públicas en infraestructura, existe un desmantelamiento de los servicios públicos, reduce los gastos de los servicios de bienestar social, quedándose sólo con las funciones de protección y seguridad y tiende a reprivatizar ciertos sectores de la economía (Pérez y Mirón, (s/f): 40).

Entonces, la reforma del Estado no es para regresar-reconociendo que antes sí lo era- a un Estado omnipresente que obstaculiza e inhibe la participación social, ni para volver al Estado tutelar que concibe a los actores sociales como menores de edad, ni para repetir los fracasos del Estado empresario que genera y nutre intereses que acababan contraponiéndose a los del conjunto social. Sí, para conformar un Estado fuerte y eficiente en el cumplimiento de sus tareas básicas, que debido a la fundamentación liberal serán las de vigilancia y control, como ejercicio responsable de la autoridad, así como la observancia legal, de acuerdo con el discurso estatal (Rebolledo, 1993: 52). Pero la mayor participación de la esfera de lo privado se restringe sólo a la empresa, y la autonomía responsable se traduce, para el caso de las clases dominadas, en sometimiento a los imperativos del capital y en destrucción de las conquistas sociales de los trabajadores.

En relación con lo económico, los cambios se dirigen a contener la inflación, tener mayor crecimiento, superávit fiscal, saneamiento financiero gubernamental, mayor inversión extranjera, predominio de exportaciones no petroleras. La estabilidad macroeconómica mediante la reducción del déficit fiscal y el sobreendeudamiento y la concertación entre los sectores es la base sobre la cual se finca el cambio estructural: la apertura comercial, la desregulación, la inversión extranjera y las desincorporaciones, y el Tratado de Libre Comercio (TLC) con Estados Unidos y Canadá, resultados que se desprenden de la reforma del Estado, pero que, en la puesta en práctica están muy lejanos de lo que se preveía. 
En términos generales se puede decir que la reforma del Estado y las políticas económicas se aglutinan en los siguientes apartados, de acuerdo con el discurso estatal, que se basan en tres estrategias: la construcción de una nueva economía, la redimensión del tamaño del Estado y la desregulación de la actividad económica modificando el marco jurídico; así se constata la imposición de una nueva estrategia de acumulación, en la cual el Estado limita su acción a la producción y a la generación de bienestar social.

Dos vertientes caracterizan la transformación del Estado: mayor presidencialismo y autoritarismo, pues es el ejecutivo el que inicia y promueve la reforma, la otra vendrá como consecuencia de los cambios; muchas son las posibles manifestaciones de la recomposición: la gradual desaparición de los poderes corporativos, la centralización del poder, el fortalecimiento del sector privado, la penetración del capital del exterior bajo una competencia más violenta, los efectos políticos que transforman los poderes relativos de los distintos grupos de la sociedad, mayor concentración y centralización del capital y una menor distribución de la riqueza.

La política social del Estado neoliberal pone a disposición del mercado funciones, prestaciones e instituciones sociales. Se trata de refundar el orden político, económico y social (moral) sobre la competencia del mercado; para ello la acción estatal debe asegurar la igualdad de oportunidades a través del imperio de la ley; pero ni el Estado, ni los particulares, quedan obligados legalmente a actuar positivamente (hacer el bien) pues se les restringiría su libertad, con ello la política social se concibe como producida por el mercado o por el Estado, privada o pública, en ese orden. Ello es así, por la posibilidad de calcular los beneficios de la provisión de servicios sociales como la educación, la atención médica, la vivienda y la seguridad social; en otras palabras, "la política social neoliberal, se define a partir de una lógica instrumental y económica en sentido estricto (Moreno, s/f: 114).

Finalmente para establecer este nuevo esquema, el Estado tiene que reestructurar otro tipo de acuerdos y pactos sociales con una renovación de compromisos de clase, sin realizar esta tarea perdería legitimidad y se cancelaría la posibilidad de desarrollar el proyecto neoliberal como un proyecto hegemónico, que él mismo impone. Es por ello que el propio Estado, en Latinoamérica fundamentalmente, 
encabeza su transformación denominándolo como proyecto de modernización, única salida a la crisis.

El Estado no supo ajustarse a límites razonables; al contrario, su crecimiento irracional, la falta de controles sociales y políticos sobre la acción de los gobernantes, la corrupción, la impunidad y la expulsión de la sociedad de cualquier participación en la conducción del asunto público son ejemplos de esa racionalidad instrumental que matiza al sistema y amenaza con colonizar el mundo de la vida, como son los valores, la educación, el lenguaje y esos procesos subjetivos que se derivan de estas relaciones, como bien indicaría Habermas.

En términos generales se establece una nueva relación entre la esfera de lo público y lo privado en la cual prevalecen los criterios de esta última para eficientar los procesos económicos y la regulación política, bajo este tamiz se critican y modifican las relaciones sociales y culturales.

\section{Las políticas educativas}

A principios de la década de los ochenta, que algunos autores llaman como la "década perdida", la política educativa de ajuste estuvo condicionada por la austeridad, el eficientismo, el uso racional de los recursos y el control para enfrentar la crisis estructural, matizada por el discurso legitimante de democratización y modernización, política caracterizada por abandono financiero y negligencia presupuestal, que debería contribuir a la estrategia de cambio nacional. Así pues, la racionalidad se tradujo en acciones de "planeación democrática" en el sentido de pretender conciliar el crecimiento con medidas para elevar la calidad.

Uno de los sectores que sufrió con mayor intensidad los efectos de esa década perdida fue el educativo, pues se vio sometido a una doble presión: recibió el impacto directo de las políticas macroeconómicas de ajuste y estabilización, las cuales se concretaron en severas restricciones al gasto educativo, provocando un deterioro en las condiciones en las cuales se establece la relación educativa como: las condiciones de estudio de la población escolar y de trabajo de los profesores. Las restricciones presupuestales se tradujeron en la caída real del salario y la consecuente disminución de los niveles de bienestar de las familias, lo que afectó las condiciones de acceso y permanencia de los educandos en las instituciones escolares. 
Los cambios en la educación, durante la década de los ochenta, se determinaban por la sostenida tendencia a la contracción en el crecimiento de las oportunidades de escolarización hasta un virtual estancamiento de la matrícula, es decir, se restringe el acceso formal a la escolaridad que aunado con la baja en el gasto, produjo una diferenciación institucional que conllevaba una serie de relaciones de dependencia que reforzaron la inequidad social en la distribución del servicio (Ibarra y Soria, 1996: 317); esta tendencia se evidencia en el nivel superior a mediados de los ochenta; así, para el año escolar 1986-1987 la tasa de crecimiento de la matrícula es menor que la del aumento demográfico y el egreso de preparatoria. Entre 1982 y 1989 esas tendencias a la disminución de las posibilidades de permanencia se observan en la atención a la demanda real que se redujo del $85 \%$ al $66 \%$ en el nivel superior. Así que todo este sistema de diferenciación y exclusión institucional se legitima en nombre de la equidad.

Igualmente los recursos reales con los que contaban las universidades públicas se redujeron severamente, conllevando el deterioro de los servicios. La proporción del PIB destinada al gasto educativo bajó de $5.5 \%$ en 1982 a $3.6 \%$ en 1987 y a $3.1 \%$ en 1989 , en tanto las universidades y tecnológicos sólo captaron el $0.8 \%$ del PIB en 1980 que para 1984 descendió a $0.67 \%$. Entre 1982 y 1987 el gasto educativo disminuyó en términos reales $34 \%$. El gasto real por alumno se redujo en $40 \%$ en 1989 ; respecto a los salarios, el impacto fue inmediato y radical, para 1988 los ingresos de los maestros de todos los niveles educativos habían sufrido una reducción que oscilaba entre el 40\% y 50\% (Fuentes, 1995: 161-163).

En este momento, de acuerdo con las recomendaciones del Banco Mundial y del FMI, para estabilizar la economía, se iba implementando un nuevo proyecto educativo acorde con estos cambios económicos y políticos, el pensamiento neoliberal, ciertamente conservador, va a conformar un espacio que comienza a modificar la concepción y el papel que se asigna a la educación superior y a la universidad pública. En este sentido se intenta hacer corresponder los sistemas de formación de recursos humanos con la producción y el mercado de trabajo, tal es el caso de la calidad de la educación que al cuestionarla se asume: que se ha deteriorado, o bien, no ha existido; pero en realidad su énfasis responde al "adelgazamiento" estatal y sus reducciones presupuestales (Ramírez y Valle, 1993). 
La educación superior fue considerada como un sector no prioritario, por lo que se establecieron medidas de ajuste presupuestal y se estableció un riguroso control sobre los salarios académicos, se dejó a cada institución adaptarse a la situación restrictiva. En esta forma, las universidades fueron incorporadas a un proceso de cambio que surge de la perspectiva de la libre competencia, educación de libre comercio para el cual no estaban preparadas, según el discurso oficial debido al predominio de la docencia, la falta de coordinación institucional entre las funciones sustantivas, la existencia de órganos de planeación ineficaces, la debilidad política de sus estructuras de gobierno, la carencia de recursos, el enfrentamiento secular con las estructuras de poder; todo ello le impedía entrar de lleno a esta imposición racional. Ante esta situación, la valoración pública de los estudios decae al volverse evidente que un diploma universitario en condiciones de "masificación universitaria" y de crisis económica no ofrece las posibilidades de colocación ocupacional y movilidad social de antaño (Kent, 1996: 381).

En 1983 se propuso el Programa Nacional de Educación Superior (PRONAES 1984-1985) que, además de consolidar el funcionamiento del Sistema Nacional de Planeación Permanente de la Educación Superior, ayudó a subsanar los déficits presupuestales para atender a la demanda y evitar los conflictos, poniendo énfasis en la vinculación del financiamiento extraordinario con la evaluación. De esta manera, la Secretaría de educación Pública (SEP) tomaba nuevamente las riendas en cuanto a la orientación social que debía incorporar la universidad pública, con apoyo de la Asociación Nacional de Universidades e Institutos de Educación Superior (ANUIES), que en 1984 propone el documento "Criterios y procedimientos generales para la evaluación de la educación superior en México".

El Programa Nacional de Educación, Cultura, Recreación y Deporte 1984-1988, fijaba los lineamientos para el desarrollo de la educación superior cuya orientación era la de una "revolución educativa" y no una política de parches y remiendos; tenía como metas y acciones las de elevar la calidad de la educación a partir de la formación integral del docente, racionalizar el uso de los recursos y ampliar el acceso a los servicios educativos, vincular la educación, la investigación científica y la tecnología con el aparato productivo y regionalizar y descentralizar la educación. 
Ello se comprueba en el énfasis puesto en la política tecnológica y científica, de De la Madrid, que se concreta en el PRONDETYC, Programa Nacional de Desarrollo Tecnológico y Científico 1984-1988 y la ley para coordinar y promover el desarrollo científico y tecnológico otorgando un papel central a la Secretaría de Programación y Presupuesto. Asimismo, en 1986 fue creado el Programa Integral para el Desarrollo de la Educación Superior (PROIDES) que habría de ser la piedra angular en la búsqueda de la excelencia académica, entendida como eficiencia, eficacia, autoevaluación y racionalización, el fortalecimiento de mecanismos e instancias centrales de planeación y el mejoramiento de las condiciones financieras de las instituciones, como fue expresado en la XXII Reunión de la ANUIES en 1986.

De esta manera, la política universitaria seguirá las directrices de la política estatal, que ahora considera prioritaria la vinculación con el sector industrial del país, el impulso a la educación técnica, a las carreras tecnológicas y que incluyen una recomendación sutil, bajo la idea de contener y revertir el crecimiento de la matrícula, reducir la inscripción en las disciplinas sociales pues se considera que no inciden en la producción, declaración del propio PROIDES. Tales medidas de la política pública para la educación superior adquieren una mayor objetivación con las acciones para reorganizar el tabulador académico, explorando con ello el uso de pocos recursos para propósitos específicos en contextos de grandes necesidades salariales y presupuestales que permite incorporar ahora, y no en modo fortuito, mecanismos de evaluación para determinar la asignación de recursos financieros a las instituciones y fijar de manera desigual por exclusiónlos ingresos del personal académico como es el caso del establecimiento del SNI (Sistema Nacional de Investigadores) el 26 de julio de 1984.

Todas estas acciones de política educativa pública van fijando un sentido y contenidos para la construcción de un proyecto educativo que impone el Estado en relación con el establecimiento de la nueva estrategia de acumulación en el país, proyecto que se evidencia a partir de: la descentralización de la educación básica y la formación de maestros que confirma el intento del gobierno de De la Madrid por reformar al Estado, que queda sólo en la reorganización administrativa, pero conservaría para el gobierno federal la dirección ideológica de la educación; la determinación de un tratamiento 
homogéneo de los conflictos laborales mediante el establecimiento de topes salariales; y contención de las movilizaciones para la disciplina sindical, lo cual condujo a una aguda crisis al interior de estas organizaciones sindicales de maestros y universitarios, con secuelas tales como los conflictos en torno a la reforma de la universidad pública, en cuyo fondo se ubica el cambio de relaciones entre la universidad y el Estado.

La modernización educativa, científica y tecnológica es una parte fundamental del conjunto de políticas modernizadoras tendientes a insertar a México dentro de las corrientes de transformación económicas, políticas y sociales del mundo por una globalización económica, que no es otra situación más que los imperativos impuestos por el cambio del régimen de acumulación que ahora se regula por medio del mercado internacional y por procesos intensivos de reproducción capitalista, por lo que ahora la concepción de planeación se vislumbra como un proceso permanente y sistemático que requiere no sólo de evaluación y autoevaluación para perfeccionar sus políticas y vigilar el cumplimiento de las funciones de la educación superior, sino de elaborar programas reguladores para alcanzar las metas educativas, así el Estado se va convirtiendo en un Estado vigilante y evaluador.

Como síntesis, se ha considerado al sexenio de Miguel de la Madrid como deconstructor de la educación superior, interpretado como una política que limpió el terreno para implantar un nuevo modelo acorde con los requerimientos del cambio de estrategia de acumulación y la transformación del Estado. Modelo que podría resumirse en tres grandes apartados: redefinición del uso de los recursos y el financiamiento; la evaluación y lo que se entiende por necesidades sociales como necesidades del aparato productivo; y creación de una nueva fuerza de trabajo flexibilizada, la visión de la escuela como empresa eficiente y la educación modelada por el mercado. Así, la modernización de la educación, con acento en la calidad de la misma, se reduce a la depreciación presupuestal y al control salarial, dejando a que cada institución se maneje a su modo, reflejando las limitaciones de una escasez desigualmente distribuida.

Ya desde 1988, con el documento presentado por el IEPES, Instituto de Estudios Políticos, Económicos y Sociales del PRI, sobre el tema de la educación superior, se reconocen, en el discurso oficial, 
los niveles deficientes de calidad académica, reclamo de diversos sectores: empresariales y sociales, por lo que es obligación del Estado promover una educación superior de calidad, es decir, en ello queda implícita la idea de adecuar la educación al aparato productivo y a la competitividad del mercado, utilizando a la práctica de la evaluación como estrategia preponderante.

\section{El problema de la calidad}

Existe una transformación económico-social a partir de la larga crisis en el país, con base en un cambio de las estrategias de acumulación que el Estado ha denominado como el proyecto nacional modernizador fundamentado en el "liberalismo social" y que le implica, necesariamente, su propia reforma, pero que no deja de reforzar su control en la educación propiciando un nuevo modelo y así buscar un cambio en la deteriorada función educativa para arribar, según el discurso estatal, a la calidad y excelencia académicas con el fortalecimiento de mecanismos e instancias centrales de planeación y la diferenciación de las condiciones financieras de las instituciones que sean acorde con estos cambios. Otro de los factores que incide sobre el aumento de exigencias hacia la educación es la intensificación de los procesos de integración global, la apertura de la economía y la revolución de la microelectrónica.

Para darle un sentido más claro y ponerla en práctica de tal manera que se concrete para los diversos niveles e instituciones de educación, Salinas de Gortari aclara en su discurso del 15 de mayo de 1989 lo que entiende por modernización:

...la modernización que propone el gobierno responde a los requerimientos de la inserción de la economía nacional en el mercado internacional(...)la nueva división internacional del trabajo supone una reconversión industrial...tal modernización presupone procesos de recalificación del trabajo manual e intelectual, en correspondencia con las nuevas necesidades de producción, marcada por la automatización y la acelerada transformación tecnológica (Salinas de Gortari, 1989).

En esta forma, la política educativa empieza a tomar una forma más precisa, acorde con los requerimientos de la política económica neoliberal: la modernización de la educación. Se sustenta en dos lineamientos: la crítica severa al desempeño de las instituciones de educación superior, específicamente la universidad pública, por su ineficiencia y baja calidad y sobre la base del proyecto deconstructor 
fundamentado en las restricciones presupuestales que conlleva la diferenciación institucional; con las reformas normativas correspondientes, como al artículo tercero constitucional y la nueva Ley General de Educación (1993). En este sentido para la universidad pública se ha buscado la adecuación a los designios del mercado, la empresa privada y la racionalidad basada en la competencia y la rentabilidad, partiendo de una crítica severa sobre su desempeño por la ineficiencia y la baja calidad, con ello se reivindica a la universidad privada, como el modelo a seguir.

Sin embargo, este modelo neoliberal no tiene entre sus prioridades la educación como tal, ya que no precisa de una mayor escolaridad de la población trabajadora; según el XI Censo General de Población y Vivienda (marzo 1990) el 12.44\% de la población mayor de 15 años es analfabeta y el $29.31 \%$ de ese mismo rango de edades no tienen la primaria completa. Aun más, en agosto de 1994, el titular de la SEP, Pescador Ozuna, afirmó que 25 millones de trabajadores, el $80 \%$ de la PEA, no concluyeron su educación primaria o son analfabetas y sólo 4 millones tienen como escolaridad máxima la secundaria, mientras que los técnicos o "profesionales medios" apenas suman 279 mil personas (Ornelas, 1995: 10).

También se recordará que, a partir de 1983, los recortes en los presupuestos de las universidades llevaron a un rápido deterioro del sector público de la educación. Desde entonces, la demanda por parte de grupos urbanos medios viene desplazándose hacia universidades privadas, que sólo evidencia la crisis de financiamiento del Estado. Así, conviene señalar que, a lo largo de los años ochenta, la crisis físcal del Estado y la consecuente caída de los recursos asignados a las universidades se tradujeron en una pérdida de calidad en el sector público, expresada en bajos salarios, falta de materiales didácticos y de laboratorio, así como en el deterioro e insuficiencia de instalaciones escolares, entre otros aspectos.

El Programa para la Modernización Educativa 1989-1994, que indica un diagnóstico de los diversos niveles educativos y las acciones para enfrentar los problemas que se citan como falta de eficiencia, de formación de maestros y de racionalización de recursos, en los cuales se objetivan para la educación las políticas de liberación económica y los mecanismos de regulación por el mercado, la desregulación y privatización, en la idea de la productividad per se, acordes con el 
modelo neoliberal altamente concentrador de la riqueza y del ingreso; y el papel histórico e irrenunciable del Estado como educador, pues asumirá la conducción de tal proceso.

Este programa establece como primer objetivo para la educación superior el "mejoramiento de la calidad" para formar profesionales que requiere el desarrollo nacional; contiene tres ejes centrales que permean su discurso: la descentralización, una nueva relación Estado-sociedad y la eficacia. Para objetivarlos se plantean siete retos: la descentralización, el rezago, el crecimiento demográfico, el cambio estructural, la vinculación entre los ámbitos escolar y productivo, el avance científico tecnológico y la inversión educativa; aunque no indica cómo realizarlos, sí establece ciertas estrategias generales como: la selectividad en el financiamiento, la diversificación de las fuentes de ingresos, los sistemas de evaluación, la implantación de estímulos a la productividad y la comercialización de los productos académicos (Del Campo, 1991: 111-112).

La universidad pública dejó de ser valorada y reconocida para ser profundamente cuestionada, criticada y presupuestalmente abandonada; los elementos que se utilizaron en la crítica por su ineficiencia fueron los bajos índices de eficiencia terminal, deficiencia para obtener un empleo que se consideró mecánicamente como indicador de la baja calidad, crítica muy a la ligera pues no se analizan los efectos de una economía en contradicción, neoliberal, que tiene como supuesto el desempleo para abatir los salarios y mantener los procesos de acumulación del capital. Así se llega a la conclusión de que las universidades deben de ser autofinanciables, construyendo un discurso tecnocrático que emplea el lenguaje elaborado desde lo privado: calidad, eficiencia, productividad en donde el sentido social de la educación se supedite al logro de las metas que se derivan del capitalismo "voraz" del neoliberalismo (Díaz, 1995: 37-40).

El discurso de las políticas del desprestigio de las universidades públicas favorece la privatización de la educación y la conformación de un imaginario social en el que se atribuyen calidad y excelencia académicas a las universidades privadas, certificadas por universidades extranjeras, como paradigma a partir del cual se significa la idea de calidad. No habría que olvidar que el sistema de pago por méritos se constituye bajo la influencia de aquello que desde 1908 se aplica en Estados Unidos denominado Merit Pay (Díaz, 1995: 
34-35,42). Contra estos parámetros se evalúa la educación pública, dejando de lado el análisis del devenir institucional y la consideración de la generación de procesos y productos que han incidido en la sociedad; baste mencionar en cuáles instituciones, las universidades públicas, se ha desarrollado la investigación de punta.

En 1990 existían 43 universidades públicas, representando el 12\% de las instituciones de educación superior; pero aún albergan a la mayor parte de la población estudiantil: el 68\% (Kent, 1993: 353). De ahí su importancia y la necesidad, en términos del proyecto neoliberal, de su transformación, pues todavía siguen formando los profesionistas para el mercado laboral y productivo, que se insertarán como maquila intelectual en una economía competitiva, debido a los procesos de integración económica desigual. El crecimiento de la matrícula, en estas instituciones, ha ido creando, en forma atropellada, un mercado académico nacional. El desarrollo del posgrado ha sido fuerte pero desigual, de ahí que se estableciera el Programa Nacional de Posgrado en ese mismo año.

Las líneas políticas del proyecto de modernización universitaria subrayan una mejora en la calidad y pertinencia social de la educación; prescriben cuatro medios estratégicos centrales para ello: evaluación, competitividad por el financiamiento, apertura y vinculación de las instituciones educativas con el sector productivo y finalmente las reformas organizativas y administrativas.

ANUIES presentó a la Secretaría de Educación Pública, en abril de 1989, el documento "Declaraciones y aportaciones de la ANUIES, para la modernización de la educación superior", producto de la consulta a las instituciones y realizada a petición del presidente de la república y aprobado por la Asamblea General de la Asociación. En este documento se expresa una concepción de la calidad de la educación superior y una estrategia para su mejoramiento:

...la calidad no sólo hace a la mejora en la eficiencia o eficacia en el logro de los productos deseados, sino también a la definición cualitativa de esos mismos deseos, expresados en objetivos o finalidades de la educación, en congruencia con lasnecesidades o proyectos nacionales. El concepto decalidad denota una acepción de cualificación o de calificación dentro de una escala valorativa, pero también implica opciones, preferencias o intereses en un espectro de posibilidades. Una determinada calidad de la educación está condicionada por sus referentes contextuales: políticos, económicos y socioculturales. La calidad actual del sistema de educación superior es la resultante de un proceso histórico en el que se han conjugado actores tanto endógenos como exógenos a las 
instituciones, que han afectado seriamente, en los últimos años, las posibilidades de desarrollo académico de las instituciones y, por tanto, la calidad de sus procesos y resultados. Las instituciones agrupadas en la ANUIES consideran que preguntarse por la calidad es, de alguna manera, un ejercicio de evaluación, de valoración; es preguntarse por el sentido y la significación individual y social de las actividades y las prácticas, por la congruencia con los fines de la educación y por la trascendencia social e histórica de los procesos y acciones académicas... La calidad es también una finalidad que se busca alcanzar y mediante acciones planeadas; es un desideratum que contribuye al crecimiento y desarrollo de las instituciones. La calidad es un objetivo que se debe buscar de manera constante, pero que nunca puede alcanzarse en forma definitiva, puesto que los procesos educativos son dinámicos y cambiantes, como lo es la sociedad en que están insertos. Para elevar la calidad de la educación superior es necesario crear o consolidar las condiciones institucionales que hagan posible el adecuado cumplimiento de sus funciones. Han de hacerse planteamientos integrales de carácter institucional y no exclusivamente de índole académica. En este sentido cobra una gran importancia el ejercicio de la evaluación como medio para conocer y valorar, con precisión, el nivel de calidad de las funciones sustantivas y de los medios y condiciones disponibles, y como punto de partida para sustentar decisiones, adoptar medidas para corregir errores y rectificar rumbos o para ratificar y consolidar acciones bien encaminada (Declaraciones y aportaciones de la ANUIES para la modernización de la Educación Superior, 1989).

Éste es el basamento que permite incorporar la idea de la calidad a las instituciones de educación superior, pues pone de relieve la relación entre fines de la educación y "necesidades o proyectos nacionales", específicamente con el neoliberal, asimismo, sin que quede claro lo que se entiende por calidad de la educación, paradójicamente se afirma que nunca se alcanzará por lo dinámico del proceso, sí subraya la necesidad de valorarla o evaluarla; de estos planteamientos se deriva una crítica severa a la "universidad de masas", por la ausencia de vinculación de la educación con el sector productivo y el desarrollo científico-tecnológico, es decir, la idea de la calidad se torna en el eje de un discurso educativo que permite subordinar la enseñanza al capital e imponer criterios selectivos para su valoración. De tal modo que se imponen criterios de privatización de lo público, concentrándose, en este caso, en la universidad pública.

La pretensión de una universidad de calidad donde el principio organizativo sea la racionalidad formal y la elevación de productividad a ultranza, por medio de controles, postula la descalificación y recalificación selectiva de la fuerza de trabajo, una intensificación de la competencia y, por ende, una recomposición competitiva del mercado 
de trabajo (Gilly, 1988: 113-119). Pero también abre la puerta para hacer recaer la calidad en la formación de los docentes y postular su profesionalización, lo cual ha dado lugar a la emergencia, vigilancia y control de la figura del académico que se ha venido configurando como un nuevo sujeto de la educación superior. Docentes e investigadores que perciben remuneraciones cuyo monto no les permite dedicarse exclusivamente al trabajo académico en una sola institución debiendo asumir actividades dispares y simultáneas, el multiempleo, en varios mercados o dentro del propio mercado académico.

La política salarial gesta una vigilancia más estrecha sobre los académicos e impone una orientación de su trabajo, una mayor flexibilización en el trabajo y evita los altos costos que supone un aumento generalizado al salario y con ello a su vez, cancela la negociación sindical al no considerar los estímulos como ingresos legalmente integrados al salario. El aumento de la capacidad estatal para conducir y controlar a las instituciones de educación superior y sus académicos, encontró en la diferenciación uno de sus mejores soportes.

La cuestión de la calidad de la educación se ha estado contemplando a través de las tentativas de institucionalizar los procesos de evaluación, y ninguno se ha consolidado aún, pero tampoco se han reformulado proyectos académicos y de reorganización institucional, debido a que no puede un solo concepto de calidad, una sola valoración, un solo tipo de parámetros o simplemente porque no se define claramente, ni en el programa de modernización ni en la política, ser la meta a la que se tiene que arribar, pues no se incorpora un aspecto decisivo y clave del proceso educativo, como lo es el relativo al modelo educativo y la organización académica; es decir, el modelo de modernización educativa no incorpora los componentes que constituyen el contexto académico organizacional del proceso de enseñanza aprendizaje, por lo que se corre el riesgo de que las políticas modernizadoras no alcancen el efecto deseado en consecución de la calidad, pues olvidan situarla bajo sus referentes contextuales.

Debido a que tal proyecto ha dependido de su articulación con el proyecto social y económico, con las formas específicas de interpretarlo y concretarlo por parte del Estado, entonces se debe propiciar la competencia desigual entre las instituciones de educación superior, IES, estableciendo criterios de productividad, de evaluación, 
acreditación, como estándares mínimos de conocimientos y su verificación, baste señalar los exámenes generales de egreso de la licenciatura implementados en marzo de 1992; ahora son instancias externas las que certifican las profesiones, quitando a la universidad su función, además de optar por las medidas de solución que emanan de directrices gubernamentales, como instancia central, a las que se ajustarán (Valenti, (s/f): 119-120).

La política modernizadora plantea la urgente necesidad de elevar la calidad de la educación y ésta se concibe como pilar del desarrollo del país, para elevar la productividad; es decir, el Estado reforma y reestructura la educación con objeto de elevar su "calidad" para responder a condiciones de crisis: en la actual coyuntura, se establece a partir del modelo de desarrollo del país, de las necesidades financieras del gobierno y de las demandas de control por parte del Estado. En ese sentido, las políticas de modernización de la educación inciden en las universidades públicas para reafirmar al Estado como rector de la política educativa nacional, sin violentar su autonomía pero ajustándolas a un modelo de desarrollo neoliberal. Así, el propósito de elevar la calidad de la educación superior sólo se utiliza como una ideología para readecuar a la universidad pública a los imperativos de la nueva estrategia de acumulación que exige una formación orientada a la productividad y a la competitividad.

Como se dijo, las líneas políticas del proyecto de modernización universitaria prescribe cuatro medios estratégicos centrales: evaluación, competitividad por el financiamiento, apertura y vinculación de las instituciones educativas con el sector productivo y finalmente las reformas organizativas y administrativas. En forma más precisa, las acciones estratégicas dependen de los medios centrales que derivan en: una evaluación externa, solicitada por la SEP al Consejo Internacional para el Desarrollo Educativo CIDE coordinado por Coombs, quien entregó un reporte de evaluación en 1991, denominado "Recomendaciones para el mejoramiento de la calidad educativa", tomándolo como parámetro para implantar el nuevo modelo educativo; asimismo, se solicitaron otras evaluaciones a organismos internacionales como CRESALC en 1992, CEPAL-UNESCO 1992, OCDE 1994, o bien, se realizaron algunas evaluaciones nacionales como la de Guevara Niebla en 1992. Evaluaciones que sirvieron para justificar el discurso estatal acerca de la falta de calidad de la educación en el país. 
La evaluación institucional que se origina en 1990 se dirige en primer término al sistema de educación superior e interinstitucional, proceso que tiene lugar entre 1991 a 1993, para proseguir con la certificación profesional y la acreditación de programas de posgrado. La evaluación de los académicos se establece con el Programa Nacional de Estímulos al Desempeño Académico en febrero de 1991; más tarde se convierte en el Programa de Carrera Docente del Personal Académico en 1992. La evaluación a alumnos se concreta con el examen nacional para ingreso a licenciatura en 1995; también se ha evaluado a los trabajadores administrativos, así como los procesos de formación, investigación, planeación y administración; el establecimiento de la evaluación a los sujetos y procesos de la educación ha conducido a la competitividad por el financiamiento, ya que se establecen partidas especiales, bolsas específicas, financiamientos extraordinarios para cada tipo de actividad evaluada o por evaluar.

La vinculación con los sectores productivos ha asumido, hasta estre momento, las modalidades de asesoría, gestoría o proyectos especiales, entendiendo la vinculación en forma muy estrecha. Además se han establecido ciertas reformas organizativas y administrativas, que han permitido establecer mecanismos de control sobre el trabajo académico y el uso de los recursos financieros.

Es así como el discurso sobre "la cultura de la evaluación", en el país, nace ligado al esquema de relación entre el Estado y universidad y como concepto que engloba lo que hace una organización de evaluación paragubernamental y no autónoma, "que actúa más como un apéndice de la administración de los recursos que como un referente autónomo para el mejoramiento de la educación (Aboites, 1997: 6).

Entonces es la evaluación el dispositivo que decide tratamientos financieros diferenciados o selectivos; su finalidad es remodelar las relaciones entre universidad, Estado y aparato productivo; opera como instrumento selectivo punitivo para identificar eficiencia y niveles de productividad. Dicha estrategia se caracteriza por el manejo selectivo de los recursos financieros, y el cambio en el patrón de financiamiento para las universidades, cuyos criterios serían: las características de la planta de profesores, el establecimiento de sectores y campos disciplinarios prioritarios, el impulso diferenciado de una política de 
incentivos que tienen como base la productividad del trabajo académico, y la evaluación institucional como tópicos centrales.

Así, a partir de los años noventa, la estrategia pública de cambio dirigido encontró la manera de acceder a un mayor control sobre la universidad por la vía de desplegar las potencialidades de la mercantilización de las relaciones entre el Estado y las instituciones, "tanto cambias, tanto te doy", con sistemas de becas y estímulos a los trabajadores académicos individuales con criterios meritocráticos para obtener una parte de los ingresos y con ello amortiguar el descenso del poder adquisitivo del salario. Así, desde hace casi veinte años, a los académicos se les ha venido quitando la facultad de discutir y acordar colectivamente las iniciativas que afectan sus condiciones de trabajo y vida.

El Fondo de Modernización de la Educación Superior FOMES, creado en abril de 1991, y el surgimiento del aparato nacional de evaluación, la Comisión Nacional de Evaluación CONAEVA en 1989, Centro Nacional para la Evaluación de la Educación Superior, CENEVAL para las evaluaciones institucionales y de estudiantes, son los mecanismos institucionales para instrumentar una política basada en la evaluación; su vinculación con el financiamiento queda sustentado con el FOMES, que distribuye recursos extraordinarios para proyectos universitarios que cumplan con los requisitos impuestos de competitividad sin considerar sus condiciones, por lo que se recalifica o se descalifica a las instituciones (Aboites, 1997: 6).

De ahí que la universidad pública enfrente uno de sus mayores retos: modernizarse sin perder su esencia cultural o morir de inanición. Muchas de las universidades no han resistido la embestida de la crisis económica y política y se debaten entre la vida y la muerte; entre la privatización frente a menguados subsidios y mantener su lugar como corporación pública (Valdivia, (s/f): 176).

Así es como a través del financiamiento público que a las universidades se introducen los criterios de selectividad y eficiencia. Además, los subsidios reflejan una intrincada red de relaciones de peso y fuerza entre las universidades, y entre éstas y el Estado; expresando el entramado de intereses cristalizado en esas relaciones, es posible observar la constitución de verdaderas relaciones clientelares y de padrinazgo entre algunas instituciones (Brunner, 1996: 138). La 
condición material refuerza y profundiza las desigualdades, inequidades, antagonismos y contradicciones en la educación superior.

Desde 1991 hasta 1993, el monto de recursos extraordinarios del FOMES se incrementó en términos absolutos 323\%, al pasar de 70 millones a casi 300 millones de nuevos pesos, así se convierte en un procedimiento "de premios y castigos" presupuestales, que permite inducir cambios en la orientación de los programas de investigación y acentuar la disposición de las autoridades de centros educativos a colaborar con el proyecto gubernamental. En ese sentido se pretende instrumentar la vida universitaria desde fuera con el llamado nuevo CONACyT, 1990, intentando modificar el objeto de la misma con base en evaluaciones que tienen una lógica que se aproxima a la del mercado en dos sentidos: como instrumento correctivo para identificar fallas en el desvío de recursos como base para adoptar medidas de reestructuración, y como instrumento selectivo para identificar eficiencia y potencial de productividad de las instituciones. Pero como queda en cierta ambigüedad correctiva y selectiva, por sí misma no lleva al mejoramiento del nivel de investigación.

Así es como la entrega de fondos queda sujeta a una supervisión de planes y a una evaluación de resultados, como en cualquier institución privada. El quehacer universitario público ya no genera el derecho a recibir recursos para su desempeño, sólo genera el derecho de oportunidad para competir en la obtención de esos recursos. Tal es el caso del PROMEP, Programa de Mejoramiento del Profesorado de las Instituciones de Educación Superior (1995), que intenta una nueva reorganización integral de la educación superior, impone un "deber ser” y perfiles de lo que es la ciencia (científico-práctica) y el conocimiento, con una supervisión hasta el detalle y una evaluación que hace la entidad financiadora para la entrega de fondos.

Con esto, el PROMEP construye un patrón de uniformidad o una "diversidad oficial" de la realidad universitaria generada desde fuera y única para todo el país; borrando la diversidad propia de cada institución y clasifica de acuerdo con la competitividad: de primera, de segunda o de tercera, agravando con estos procesos la diferenciación; es decir, gesta una visión centralizadora de las dependencias que competirán externa e internamente por el financiamiento. Tal competencia incentiva que algunas instituciones hagan esfuerzos 
desproporcionados por generar programas de excelencia, a costa de los recursos necesarios para sobrevivir (Aboites, 1997: 7-10).

Sin embargo, tal política se convierte en un discurso ideológico que intenta ampliar la racionalidad técnica y la competitividad, para imponer un tipo de modelo de desarrollo modernizante y neoliberal desde fuera, para incorporarse a la división internacional del trabajo. Así pues, el objetivo de preparar para la productividad es el punto de partida para la construcción del concepto de calidad de la educación (Calvo, 1993: 6).

Pero ¿qué es lo que realmente se propone la política con utilización de la estrategia de evaluación de la educación? En realidad ha procedido de la negociación y de los compromisos entre la esfera de lo público y de lo privado, de tal modo que se pueden advertir dos momentos: el primero consiste en un traspaso del discurso de la esfera de lo privado hacia lo público, específicamente en la universidad pública; el segundo muestra el modo en que la propia esfera de lo público se reestructura a sí misma privatizando ciertos ámbitos como las instituciones de educación superior y ciertos procesos al interior de estas instituciones como serían la vinculación con la producción, la evaluación de los académicos, los financiamientos extraordinarios y la exigencia de buscar fuentes alternas, por citar algunos.

Reafirmar la selectividad acentuada en el nivel escolar, convierte a las universidades en centros más elitistas de educación superior, promueve la segmentación de la educación y una fragmentación del mercado de trabajo y la aceptación social de la idea de que ya no existen trabajos "para toda la vida", sino trabajos más o menos precarios o que se requieren periodos de recalificación con salarios reducidos o trasladados hacia el lugar donde las empresas puedan obtener mayores rendimientos, con la necesidad de desarrollar nuevos valores tales como: renuncia, aceptación, disciplina al orden jerárquico. Ello lo entiende el discurso estatal como la manera en que la educación da respuesta a las necesidades sociales, que no son otras más que las del aparato productivo, que exige la creación de una nueva fuerza de trabajo, flexibilizada, y una nueva visión de la escuela como empresa eficiente modelada por el mercado, ésta es la manera en que la educación superior se adecua, como imposición, a la estrategia de acumulación que se ha venido instaurando desde los años ochenta. 
Con estas políticas se llega a una educación superior profundamente segmentada, con apoyos diferenciados, incluso al interior de las instituciones a costa de las necesidades sociales de conocimiento y educación de las mayorías, con un régimen laboral que deteriora derechos de participación en la determinación de las condiciones de trabajo del académico y los convierte en empleados cuasi gubernamentales sin protección. Asimismo, se consolida la conducción de la universidad en manos de un reducido grupo de tecnócratas funcionarios y rectores.

De ahí que se proponga reconsiderar a la educación superior a partir de la crítica a las políticas públicas formuladas desde lo privado, por sujetos nacionales como son los empresarios y por organismos internacionales como el Banco Mundial o el Fondo Monetario Internacional, pasando por lo público para imponer un modelo de desarrollo y de sociedad, que tiene como base una racionalidad instrumental que coloniza, por medio del dinero y el poder, a la educación, colonización que se constata en la manera en que se manipula el manejo del financiamiento a las instituciones, o bien, en los modos como se imponen legalmente las acciones de la política educativa en ese amplio entretejido de relaciones que se establecen en la educación, cancelando la comunicación y el lenguaje, los valores que posibilitan los acuerdos consensuados y la intersubjetividad que matiza las relaciones sociales en su conjunto.

\section{Bibliografía}

Aboites, Hugo (1997), "El Promep: la transformación de la universidad en dependencia gubernamental", en Vientos del Sur, núm. 10, México.

Altvater, Elmar (1979), "Política económica y crisis", en Cuadernos políticos, núm. 22, octubre-diciembre, México: Editorial Era.

Boyer, Robert y Benjamín Coriat (1985), "Marx, la técnica y la dinámica larga de la acumulación", en Cuadernos Políticos, núm. 43, abril-junio, México.

Brunner, José Joaquín (s/f), "Educación en América Latina durante la década de 1980: la economía política de los sistemas", en Kent, Rollin (compilador) Los temas críticos de América Latina, México: FLACSO.

Calvo Pontón, Beatriz (1993), "El Acuerdo Nacional para la Modernización de la Educación Básica: una interpretación regional desde la frontera norte", en Cuadernos de trabajo de la Unidad de Estudios Regionales, núm.11,Cd. Juárez: Universidad Autónoma de Cd. Juárez.

Del Campo, Jesús Martín (1991), "Modernización y alternativa democrática en educación", en Politicas Estatales en Materia Educativa, Col. Estado, Sociedad y Educación, México: SNTE.

Díaz Barriga, Ángel (1995), Empleadores de universitarios. Un estudio de opiniones, México: Miguel Ángel Porrúa. 
Domenech, Antoni (s/f), Historia y crítica de la opinión pública, México.

Elkan, Walter (1975), Introducción a la teoría económica del desarrollo, México: Alianza Editorial.

Fuentes Molinar, Olac (1995), "El Estado y la educación pública en los años 80", en El Nuevo Estado Mexicano. Estado y Sociedad, México.

(s/f), "Neoberalismo en la educación", en ¿Fin de un régimen?, México.

Gilly, Adolfo (1988), Nuestra Caída de la Modernidad, México: Juan Boldo Edit.

Habermas, Jürgen (1981), Historia y crítica de la opinión pública, Barcelona: Editorial Gustavo Gilly.

Ibarra Colado, Eduardo y Víctor M. Soria Murillo (1996), "Balance y perspectivas de la modernización educativa en México. Las políticas del régimen salinista (1989-1994)", en Políticas Públicas alternativas en México, México.

Kamerman B., Sheila y Alfred Kahn (1993), La privatización y el Estado benefactor, México: Fondo de Cultura Económica.

Kent, Rollin (1996), Los temas críticos en la educación superior en América Latina, Estudios comparativos, México: FLACSO.

Kent S., Rollin (1993), "El desarrollo de políticas en Educación Superior en México: 1960 a 1990", en Políticas comparadas de Educación Superior en América Latina, Santiago de Chile:FLACSO.

Marx, Karl (1994), El capitalismo mexicano en los ochenta, México: Era.

(1978), La llamada acumulación originaria, México: Fondo de Cultura Económica.

Moreno, Pedro H. (s/f), "Elaboración de la política social y transformación del Estado", en Reforma del Estado y políticas sociales,México.

Ornelas Delgado, Jaime (1995), "Algunos efectos sociales del neoliberalismo en México", en Papeles de Población,núm.8, julio-septiembre, México: CIEAP/UAEM.

Pérez, Germán y Rosa Ma. Mirón (1993), "Keynes: racionalidad y crisis en el Estado contemporáneo", en Estudios políticos, México.

Ramírez, Rosa Ma. y Maximiliano Valle (1993), Las políticas públicas para la educación, México: (mimeo).

Rebolledo, Juan (1993), La Reforma del Estado, México: Fondo de Cultura Económica.

Valdivia, Luis Miguel (s/f), "Problemática universitaria en México y América", en Políticas Estatales en Materia Educativa, México.

Valenti, Giovanna (s/f), "Un análisis del Programa de Modernización Educativa", en Políticas Estatales en Materia Educativa, México.

Varela Petito, Gonzalo (s/f), "Las políticas públicas en el desarrollo actual de América Latina”, en Reforma del Estado y políticas sociales, México. 